\title{
Development of a Drosophila cell-based error correction assay
}

\author{
Jeffrey D. Salemi ${ }^{1}$, Philip T. McGilvray ${ }^{1}$ and Thomas J. Maresca ${ }^{1,2}$ * \\ ${ }^{1}$ Biology Department, University of Massachusetts, Amherst, MA, USA \\ ${ }^{2}$ Molecular and Cellular Biology Graduate Program, University of Massachusetts, Amherst, MA, USA
}

\section{Edited by:}

Samuel F. Bakhoum, Geisel School of Medicine at Dartmouth, USA

\section{Reviewed by:}

Matthias P. Wymann, University of Basel, Switzerland

Helder Maiato, University of Porto,

Portugal

*Correspondence:

Thomas J. Maresca, Biology

Department, 436B Morrill 4 South,

611 North Pleasant Street, University

of Massachusetts Amherst, Amherst, MA 01003, USA

e-mail: tmaresca@bio.umass.edu
Accurate transmission of the genome through cell division requires microtubules from opposing spindle poles to interact with protein super-structures called kinetochores that assemble on each sister chromatid. Most kinetochores establish erroneous attachments that are destabilized through a process called error correction. Failure to correct improper kinetochore-microtubule (kt-MT) interactions before anaphase onset results in chromosomal instability (CIN), which has been implicated in tumorigenesis and tumor adaptation. Thus, it is important to characterize the molecular basis of error correction to better comprehend how CIN occurs and how it can be modulated. An error correction assay has been previously developed in cultured mammalian cells in which incorrect kt-MT attachments are created through the induction of monopolar spindle assembly via chemical inhibition of kinesin-5. Error correction is then monitored following inhibitor wash out. Implementing the error correction assay in Drosophila melanogaster S2 cells would be valuable because kt-MT attachments are easily visualized and the cells are highly amenable to RNAi and high-throughput screening. However, Drosophila kinesin-5 (Klp61F) is unaffected by available small molecule inhibitors. To overcome this limitation, we have rendered S2 cells susceptible to kinesin- 5 inhibitors by functionally replacing Klp61F with human kinesin5 (Eg5). Eg5 expression rescued the assembly of monopolar spindles typically caused by Klp61F depletion. Eg5-mediated bipoles collapsed into monopoles due, in part, to kinesin-14 (Ncd) activity when treated with the kinesin-5 inhibitor S-trityl-L-cysteine (STLC). Furthermore, bipolar spindles reassembled and error correction was observed after STLC wash out. Importantly, error correction in Eg5-expressing S2 cells was dependent on the well-established error correction kinase Aurora B. This system provides a powerful new cell-based platform for studying error correction and CIN.

Keywords: error correction, kinesin-5, aurora B kinase, kinetochore, spindle, Drosophila

\section{INTRODUCTION}

During cell division, correct segregation of the genome requires that replicated chromosomes become bioriented, with each sister chromatid attached to dynamic microtubules from opposite spindle poles. The interaction between chromosomes and microtubules is mediated by a protein complex, called the kinetochore, which assembles on the centromeres of each sister chromatid as the cell prepares to divide. The kinetochore is comprised of $\sim 100$ known proteins that are present in multiple copies and organized into a conserved molecular architecture consisting of two spatially distinct electron-dense regions generally referred to as the inner and outer kinetochore (1-5). Kinetochore-microtubule (kt-MT) interactions are mediated through numerous outer kinetochore factors. The establishment and maintenance of end-on kt-MT attachments requires the KMN (KNL1/Blinkin, Mis12 complex,

Abbreviations: ABK, aurora B kinase; CIN, chromosomal instability; DMSO, dimethyl sulfoxide; GFP, green fluorescent protein; Klp61F, kinesin-like-protein 61F (Drosophila kinesin-5 motor); kt-MT, kinetochore-microtubule; Ncd, non-claret disjunction (Drosophila kinesin-14 motor); RepEg5 cells, replacement Eg5 cells; SEM, standard error of the mean; STLC, S-trityl-L-cysteine.
Ndc80 complex) network while mature end-on attachments are reinforced through recruitment of the microtubule-binding Ska1 complex to bioriented kinetochores (6-10).

Biorientation is the ideal chromosomal configuration but it is by no means a default state. In fact, a vast majority of chromosomes in mammalian oocytes establish multiple incorrect kt-MT interactions before achieving biorientation (11). Cells have evolved an essential network of regulatory components that correct flawed interactions between kinetochores and spindle microtubules. The process by which improper kt-MT attachments are selectively destabilized is called error correction. The best characterized error correction regulator is Aurora B kinase (ABK) (12). Prior to anaphase, $\mathrm{ABK}$ is highly enriched in the centromeric chromatin that underlies the inner kinetochore. The microtubule-binding activities of both the KMN network and the Skal complex are negatively regulated by ABK-mediated phosphorylation (13-17). At improperly attached kinetochores, it is postulated that attachment factors are positioned close enough to ABK to become phosphorylated thereby reducing their affinity for microtubules $(16,18)$. ABK inhibition leads to a prevalence of incorrect interactions called syntelic attachments where each sister kinetochore is attached 
to the same spindle pole (19-21). Therefore, erroneous kt-MT interactions are commonplace but typically corrected by ABK.

The ability to visualize the conversion of erroneous kt-MT attachments to bioriented attachments in living cells would provide valuable insight into the error correction process. Indeed, a live-cell error correction assay was previously developed in mammalian cells (21). In the established assay, incorrect kt-MT attachments are induced by chemical inhibition of mammalian kinesin-5 (Eg5). Since Eg5 pushes spindle poles apart, inhibiting kinesin-5 motor activity leads to monopolar spindle assembly and results in many sister kinetochores becoming syntelically attached to the single spindle pole. Small molecule inhibitors of Eg5 can be "washed-out" to reverse their effects and during the subsequent reorganization from monopole to bipole incorrect attachments are corrected in an ABK-dependent manner.

Drosophila melanogaster S2 cells have proven to be an excellent model for studying mitotic spindle assembly and kinetochore function. The fact that Drosophila have only four chromosomes makes visualization of kt-MT attachments considerably easier than in other model cell types such as HeLa that have $>100$ kinetochores. Although S2 cell lines are not typically diploid (22) and tend to become tetraploid through passaging (23), the number of chromosomes when compared to HeLa cells still yields distinct advantages for visualization. Furthermore, S2 cells are exceedingly amenable to high-throughput whole-genome RNAi screening $(24,25)$. Unfortunately, the error correction assay cannot be applied to S2 cells because Klp61F (D. melanogaster kinesin-5) is unaffected by kinesin-5 inhibitors such as monastrol and $S$ trityl-L-cysteine (STLC) (26-28). Rendering Drosophila S2 cells susceptible to Eg5 inhibitors would overcome these limitations and provide a new cell-based tool for examining error correction and CIN.

\section{MATERIALS AND METHODS CELL CULTURE}

Drosophila S2 cells were cultured at $24^{\circ} \mathrm{C}$ in Schneider's media (Life Technologies) supplemented with $10 \%$ heat inactivated fetal bovine serum (Life Technologies) and $0.5 \times$ antibiotic-antimycotic cocktail (Life Technologies).

\section{GENERATION OF S2 CELL LINES}

The Homo sapiens Eg5 (kinesin-5) gene was amplified from pCS2EGFP-Eg5 (gift of Patricia Wadsworth, UMASS, Amherst) with a $5^{\prime} K p n I$ site and $3^{\prime}$ SpeI site. The resulting product was inserted into the multiple cloning site of a pMT/V5 His-B vector (Invitrogen) containing the mCherry gene and Blasticidin resistance marker. Human Eg5-mCherry DNA was transfected into stable Hygromycin B resistant, GFP- $\alpha$-tubulin-expressing S2 cells using the Effectene Transfection Reagent system (Qiagen), according to product directions. The transfected cells were grown in Schneider's media (Invitrogen) containing 10\% Fetal Bovine Serum (Invitrogen). After 4 days, they were transferred to a $25 \mathrm{~cm}^{2}$ flask. Cells were then grown in media containing Blasticidin S HCL (Invitrogen) at a concentration of $0.025 \mathrm{mg} / \mathrm{ml}$ until cell death ceased. At that point cells were maintained in media containing no Blasticidin. Eg5-mCherry was induced by adding $500 \mu \mathrm{M} \mathrm{CuSO}_{4}$ to the media 6-18 h before an experiment.

\section{PRODUCTION OF dsRNAs}

DNA templates for Klp61F (CG9191), Ncd (CG7831), and Aurora B (CG6620) were produced to contain $~ 500$ bp of complementary sequence flanked with the T7 promoter sequence (Table S1 in Supplementary Material). dsRNAs were synthesized overnight at $37^{\circ} \mathrm{C}$ from the DNA templates using the T7 RiboMax ${ }^{\mathrm{TM}}$ Express Large Scale RNA Production System (Promega). RNAi experiments were done as previously described (29).

\section{LIVE-CELL IMAGING}

Cells were seeded onto Concanavalin A (Sigma) treated acidwashed coverslips (Corning) for $1 \mathrm{~h}$. The coverslips were assembled into rose chambers containing Schneider's media and imaged at room temperature. Cells were imaged on a Nikon TiE inverted microscope with a cooled CCD Orca R2 camera (Hamamatsu) using Nikon $100 \times 1.4$ NA Plan Apo violet corrected (VC) series differential interference contrast (DIC) and $40 \times 1.3$ NA Plan Fluor DIC objectives. Metamorph software (Molecular Devices) was used to control the imaging system as well as for image processing and analysis.

\section{IMMUNOFLUORESCENCE}

S2 cells were allowed to adhere to ConA-coated cover slips before being quickly rinsed in BRB80 and then fixed for $10 \mathrm{~min}$ in $10 \%$ paraformaldehyde. Cells were then permeabilized for $8 \mathrm{~min}$ in $1 \times$ PBS $+1 \%$ Triton, washed $3 \times$ in $1 \times$ PBS $+0.1 \%$ Triton, and blocked for at least $30 \mathrm{~min}$ in Boiled Donkey Serum. Primary antibodies were diluted into Boiled Donkey Serum: anti-phosphoH3 Serine 10 (Abcam) at 1:20,000, DM1 $\alpha$ (anti- $\alpha$ Tubulin antibody, Sigma-Aldrich) at 1:1000, and anti-Ndc80 (generated by Thomas J. Maresca) at 1:100. All appropriate secondary antibodies (Jackson ImmunoResearch) were diluted 1:200 in Boiled Donkey Serum. Cells were treated with DAPI (1:100) and sealed in mounting media containing $20 \mathrm{mM}$ Tris (pH8.0), 0.5\% N-propyl gallate, and $90 \%$ glycerol.

\section{WESTERN BLOTTING}

A total of $10 \mu \mathrm{g}$ of protein was loaded into a 10\% SDS-PAGE gel, run out, and transferred to PVDF membrane (BioRad) in transfer buffer containing $10 \%$ methanol. All antibodies were diluted in TBS with $0.1 \%$ Tween and $5 \%$ milk. The membrane was first incubated with anti-Klp61F serum (gift of Jonathan Scholey, UC, Davis) at 1:500 and DM1 $\alpha$ (anti- $\alpha$ Tubulin antibody) (SigmaAldrich) at 1:1000 and then probed with anti-Human polyclonal Eg5 antibody (Novus Biologicals, gift of Patricia Wadsworth, UMASS Amherst) at 1:500. Rabbit and mouse HRP secondary antibodies (Jackson ImmunoResearch) were used in conjunction with their respective primaries and imaged with a GBox system controlled by GeneSnap software (Syngene).

\section{RESULTS \\ HUMAN Eg5 LOCALIZES NORMALLY THROUGHOUT THE CELL CYCLE IN DROSOPHILA S2 CELLS}

To investigate the behavior of human Eg5 (Hs-Kinesin-5) in Drosophila, a stable Drosophila S2 cell line expressing GFP- $\alpha$ tubulin and Eg5-mCherry under the control of a copper-inducible promoter was generated. During interphase, Eg5 is excluded from 


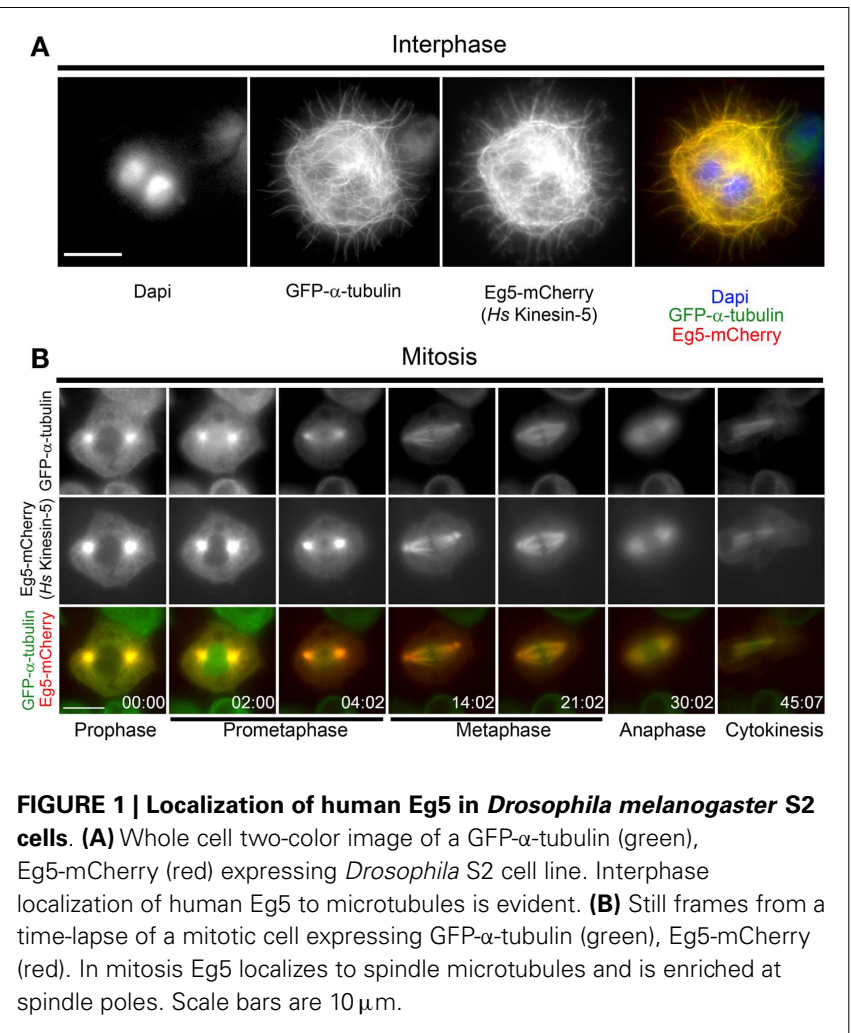

the nucleus and associates with microtubules (30) while in mitosis the motor localizes to spindle microtubules and is enriched at spindle poles (31). Eg5 expression was induced overnight with copper sulfate and mCherry fluorescence was visualized in both live and fixed cells. Exogenous Eg5 associated with microtubules in interphase cells (Figure 1A). While the interphase microtubule localization of human Eg5 in S2 cells could be attributed, in part, to Eg5 over-expression, it may also reflect an underappreciated role for Eg5, which is assumed to be a mitotic kinesin, in interphase. In mitosis, Eg5 became highly enriched at centrosomes in prophase and remained associated with the centrosomes through the entirety of mitosis (Figure 1B; Movie S1 in Supplementary Material). Following nuclear envelope breakdown in prometaphase, Eg5-mCherry associated with the microtubules of the assembling spindle and by metaphase Eg5 was enriched near the spindle poles. Eg5 remained on midzone microtubules through anaphase and localized at the midbody during cytokinesis (Figure 1B; Movies S1 and S2 in Supplementary Material). Since Eg5-mCherry localized normally in both interphase and mitotic Drosophila S2 cells, the functionality of the motor in S2 cells was next investigated.

\section{HUMAN Eg5 RESCUES KNOCKDOWN OF DROSOPHILA KINESIN-5}

Klp61F is the Drosophila kinesin-5 family member and homolog of Eg5 (32). While Klp61F exhibits significant homology to human Eg5 in its motor domain, the remainder of the protein is highly divergent and; interestingly, Klp61F is not sensitive to chemical inhibitors that disrupt Eg5 function across a range of species (26-28). Furthermore, to our knowledge, the

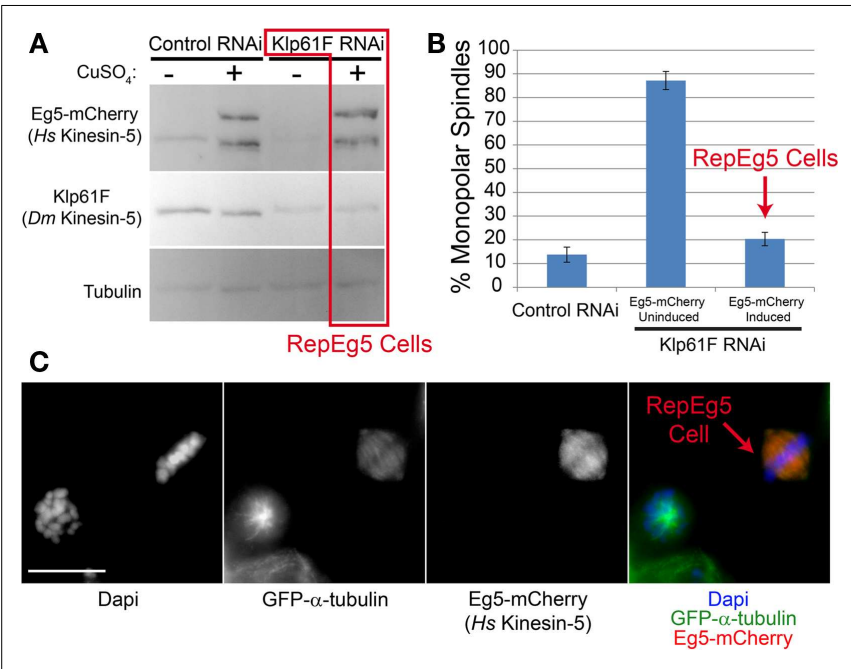

FIGURE 2 | Human Eg5 rescues depletion of the Drosophila kinesin-5 family member KIp61F. (A) Western blot analysis of cell lysates prepared from induced and uninduced; control and KIp61F RNAi-treated cells blotted with anti-Eg5 to monitor induction of Eg5-mCherry, anti-KIp61F to confirm depletion of the motor and anti- $\alpha$-tubulin as a loading control. Cells depleted of KIp61F and with Eg5-mCherry induced are referred to as RepEg5 cells. (B) Quantification of the percentage of cells with monopolar spindles in control RNAi cells versus Klp61F-depleted Eg5-mCherry induced and uninduced cells. (C) An example of a field of view containing a RepEg5 cell with a bipolar spindle next to a Klp61F-depleted cell that is not expressing Eg5-mCherry and has a monopolar spindle. In the merged image GFP- $\alpha$-tubulin is shown in green, Eg5-mCherry in red, and DAPI-stained DNA in blue. Error bars are standard error of the mean (SEM). Scale bar is $10 \mu \mathrm{m}$.

ability of human Eg5 to complement Klp61F function has never been investigated. Thus, the capacity of Eg5-mCherry to rescue Klp61F depletion in S2 cells was tested. In the absence of Klp61F, Drosophila cells fail to assemble bipolar spindles and instead generate monopoles $(33,34)$. The same was true in uninduced Eg5-mCherry expressing cells as $\sim 80 \%$ of mitotic cells assembled monopoles following depletion of Klp61F by RNAi (Figures 2A,B). To the contrary, nearly $\sim 90 \%$ of mitotic cells expressing Eg5-mCherry assembled bipolar spindles following induction (Figure 2B). After induction, Eg5-mCherry expression, which was confirmed by western blot analysis (Figure $2 \mathrm{~A}$ ), varied on a cell-by-cell basis with some cells exhibiting undetectable mCherry fluorescence. The variation in expression levels served as an internal control given that Klp61F-depleted cells without Eg5 assembled monopoles while nearby Eg5-mCherryexpressing cells nearly always built bipolar spindles (Figure 2C). Thus, Eg5-mCherry rescues Klp61F depletion in Drosophila S2 cells. Henceforth, Klp61F-depleted cells that express detectable levels of Eg5-mCherry will be referred to as Replacement Eg5 (RepEg5) cells.

\section{RepEg5 CELLS ARE SENSITIVE TO CHEMICAL INHIBITION OF Eg5}

Chemical inhibition of Eg5 results in monopolar spindle assembly (35). It has been reported that the widely used small molecule Eg5 inhibitors STLC and monastrol do not affect $\operatorname{Klp} 61 \mathrm{~F}(26,28)$. 


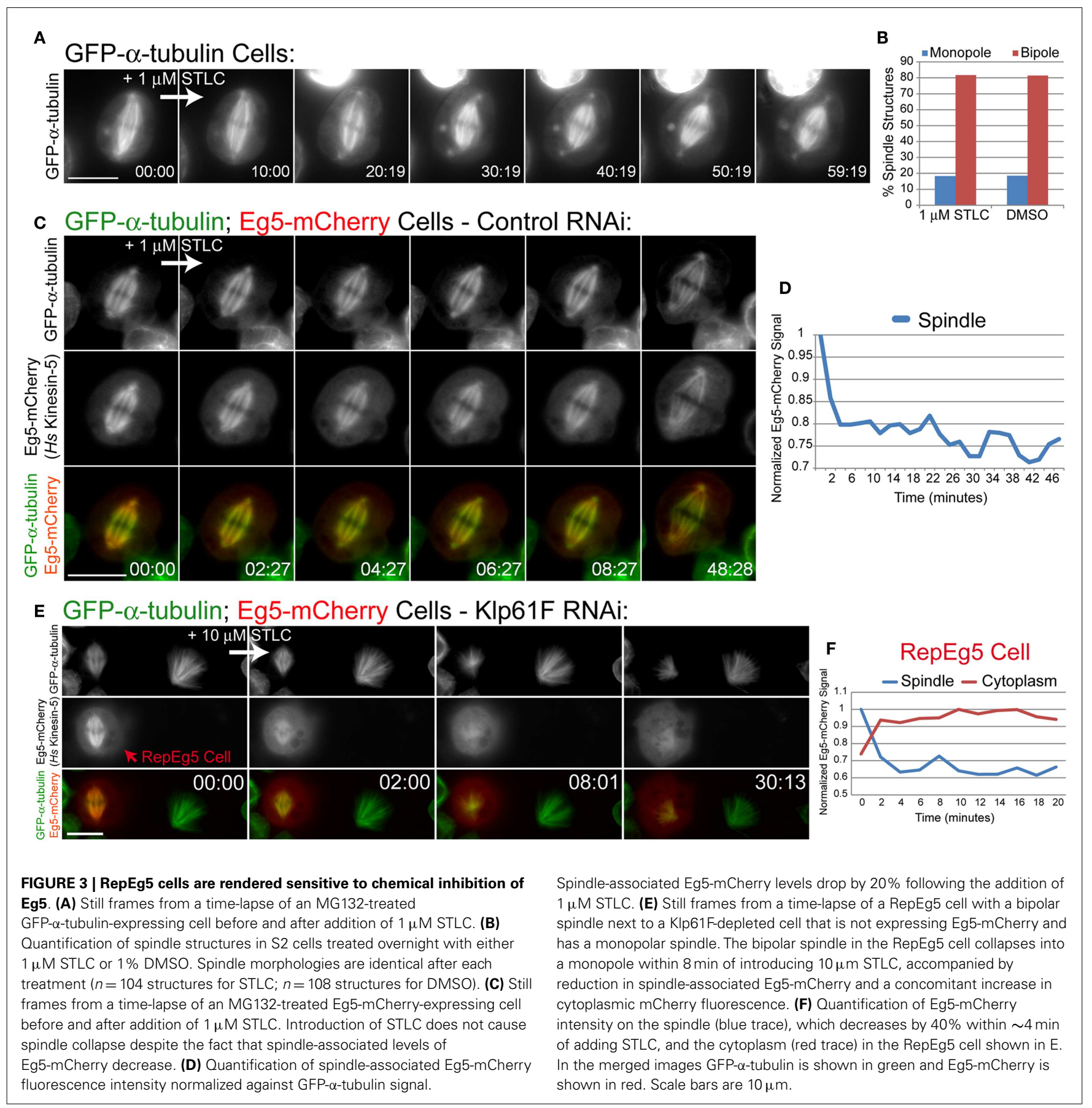

Accordingly, treatment of GFP- $\alpha$-tubulin-expressing cells with the inhibitor S-trityl-L-cysteine (STLC) did not cause spindle collapse or assembly of monopoles (Figure 3A; Movie S3 in Supplementary Material). Furthermore, spindle morphologies in cells subjected to overnight STLC treatment were identical to those in cells treated overnight with DMSO (Figure 3B). Spindle morphologies were analyzed by observing the organization of the chromosomes in mitotic cells as well as spindle pole positioning. Cells containing spindle poles that were within several microns of each other and that had splayed chromosomes arranged at the periphery of the microtubule array were deemed to have monopolar spindle. Cells with clearly separated spindle poles located on either side of a linear arrangement of chromosomes were considered to possess bipolar spindles. These data further demonstrate that STLC does not inhibit Klp61F activity.

To determine whether S2 cells were permeable to STLC, spindle-associated Eg5-mCherry fluorescence was measured following addition of the inhibitor to control RNAi-treated cells. STLC inhibits the microtubule-stimulated ATPase activity of Eg5 and causes spindle-associated Eg5 levels to drop and become 
diffusive in cells $(36,37)$. Similarly, addition of $1 \mu \mathrm{M}$ STLC to Eg5-expressing S2 cells resulted in a $20 \%$ decrease in Eg5mCherry spindle fluorescence within 2 min of introducing the drug (Figures 3C,D; Movie S4 in Supplementary Material). The observed reduction in mCherry fluorescence revealed that STLC can rapidly cross the plasma membrane in S2 cells and that STLC treatment causes a fraction of Eg5 to dissociate from spindle microtubules even when the motor is expressed in Drosophila (Figure 3D). Despite the fact that the exogenous Eg5-mCherry was evidently targeted by STLC, the presence of endogenous Klp61F prevented STLC-induced spindle collapse in control RNAi cells (Figure 3C; Movie S4 in Supplementary Material). The effects of STLC in Eg5-mCherry expressing cells following depletion of Klp61F by RNAi was investigated next. As previously discussed, RepEg5 cells assembled bipolar spindles in the absence of Klp61F (Figures 2C and 3E). Importantly, bipolar spindles in RepEg5 cells collapsed into monopoles within $10 \mathrm{~min}$ of introducing STLC (Figure 3E; Movie S5 in Supplementary Material). Spindleassociated and cytoplasmic Eg5-mCherry fluorescence intensities were also quantified during STLC treatment of RepEg5 cells. In the example shown in Figure 3E; Movie S5 in Supplementary Material, as the bipole collapsed, there was a $40 \%$ reduction in spindle-associated Eg5 and a concomitant increase in cytoplasmic fluorescence within 10 min of introducing STLC (Figure 3F). Thus, RepEg5 S2 cells were successfully rendered sensitive to chemical inhibition by STLC.

\section{STLC-INDUCED SPINDLE COLLAPSE IN RepEg5 CELLS REQUIRES THE MINUS END-DIRECTED DROSOPHILA KINESIN-14 MOTOR Ncd}

Spindle collapse following kinesin-5 inhibition is mediated by opposing forces that are generated by minus end-directed motors (56). Dynein is the principal minus end-directed motor opposing Eg5 in vertebrates (39-42) while the motor Ncd (kinesin-14) fills this role in Drosophila $(29,33,34,38,43)$. The contribution of
Ncd to the STLC-induced spindle collapse seen in RepEg5 cells was; therefore, investigated. In agreement with previous observations in Drosophila cells $(34,44,45)$, Ncd knockdown resulted in the assembly of spindles with unfocused poles (Figure 4A). Addition of STLC to control RNAi-treated cells caused dissociation of Eg5 from spindle microtubules and monopolar spindle assembly in more than $80 \%$ of RepEg 5 cells (Figures 4 A,B). There was a fourfold reduction in the percentage of RepEg5 cells with monopolar spindles following STLC treatment in Ncd-depleted cells. In fact, $66 \%$ of the Ncd-depleted RepEg5 cells assembled bipolar spindles with unfocused poles in the presence of STLC. To be confident that the cells analyzed in these experiments were indeed STLC-inhibited, only cells with high levels of diffuse cytoplasmic Eg5 were scored for spindle morphology. Thus, Ncd activity contributes to collapsing bipoles into monopoles in STLC-treated RepEg5 cells.

\section{BIPOLAR SPINDLES REASSEMBLE FOLLOWING STLC WASH OUT}

Since inhibition of Eg5 by STLC is reversible and the drug can be washed out of the cell types in which it is effective, the response of live RepEg5 cells to STLC treatment and drug wash out was examined (Figure 5A; Movie S6 in Supplementary Material). To do so, cells were treated for 30-60 min with $10 \mu \mathrm{M}$ MG132 to delay mitotic cells in metaphase. Time-lapse imaging of metaphase cells was initiated and the starting media was exchanged with new media containing $1 \mu \mathrm{M}$ STLC between acquisition of the first and second images in the time-lapse sequence. Spindle collapse and Eg5-mCherry spindle dissociation occurred rapidly and was completed within $\sim 10$ min of adding the drug. Interestingly, unlike in mammalian cells where Eg5 activity is not required for maintaining spindle bipolarity once a bipolar spindle has assembled (46), Eg5 activity in RepEg5 cells is clearly required for both establishment and maintenance of spindle bipolarity. In this regard, spindles in RepEg5 cells behave more like those assembled in

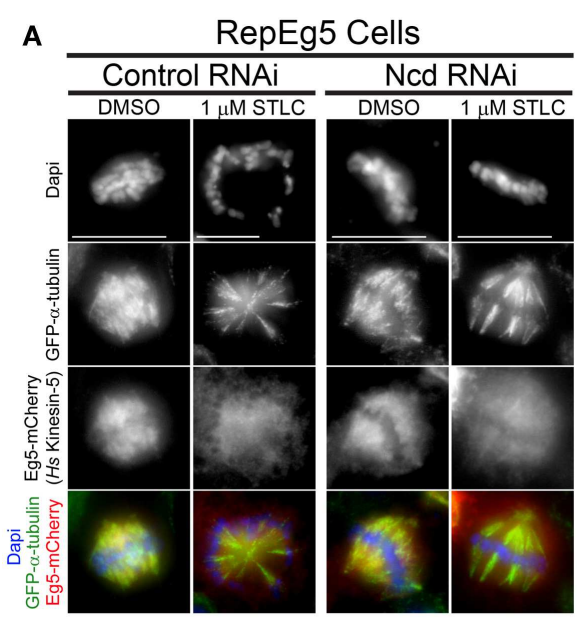

FIGURE 4 | Efficient collapse of bipolar spindles into monopoles in STLC-treated RepEg5 cells requires the minus end-directed Drosophila kinesin-14 motor Ncd. (A) Representative maximum projection images of spindle morphologies in control RNAi- and Ncd RNAi-treated RepEg5 cells in either DMSO or $1 \mu \mathrm{M}$ STLC. In the

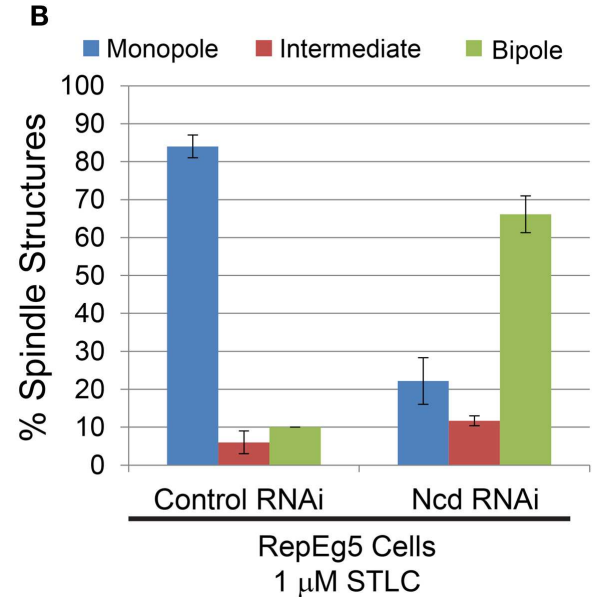

merged images GFP- $\alpha$-tubulin is green, Eg5-mCherry is red, and DAPI-stained DNA is blue. (B) Quantification of spindle structures in RepEg5 cells treated with $1 \mu \mathrm{M}$ STLC in the presence and absence of Ncd ( $n=200$ spindles in control RNAi; $n=206$ structures in Ncd RNAi). Error bars are SEM. Scale bars are $10 \mu \mathrm{m}$. 


\section{A GFP- $\alpha$-tubulin; Eg5-mCherry Cells - KIp61F RNAi:}

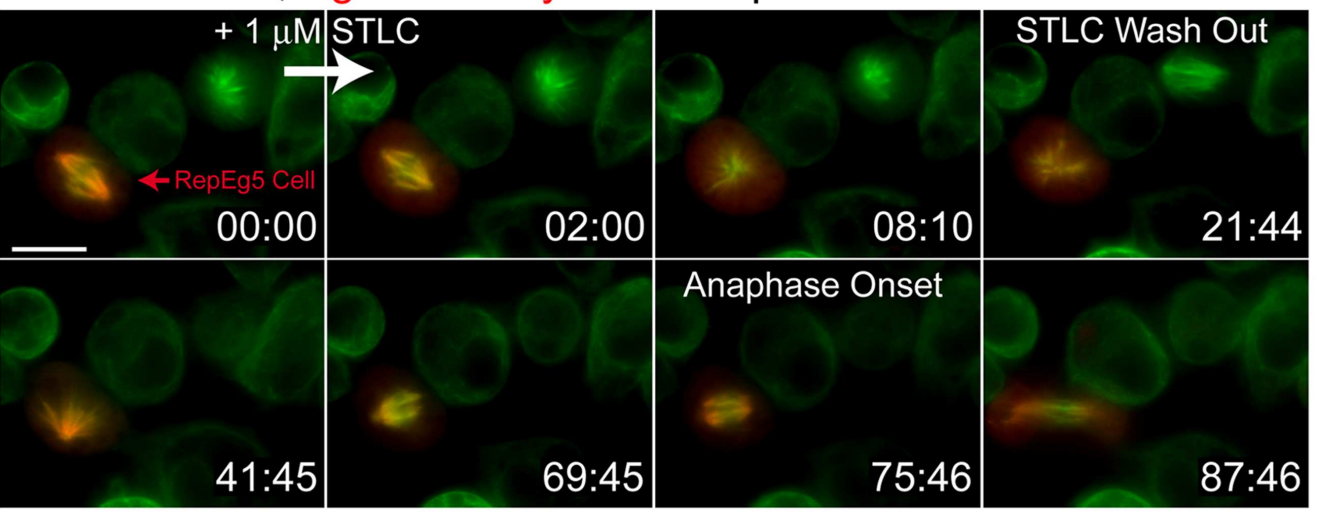

RepEg5 Cells

$1 \mu \mathrm{M}$ STLC Treatment

B

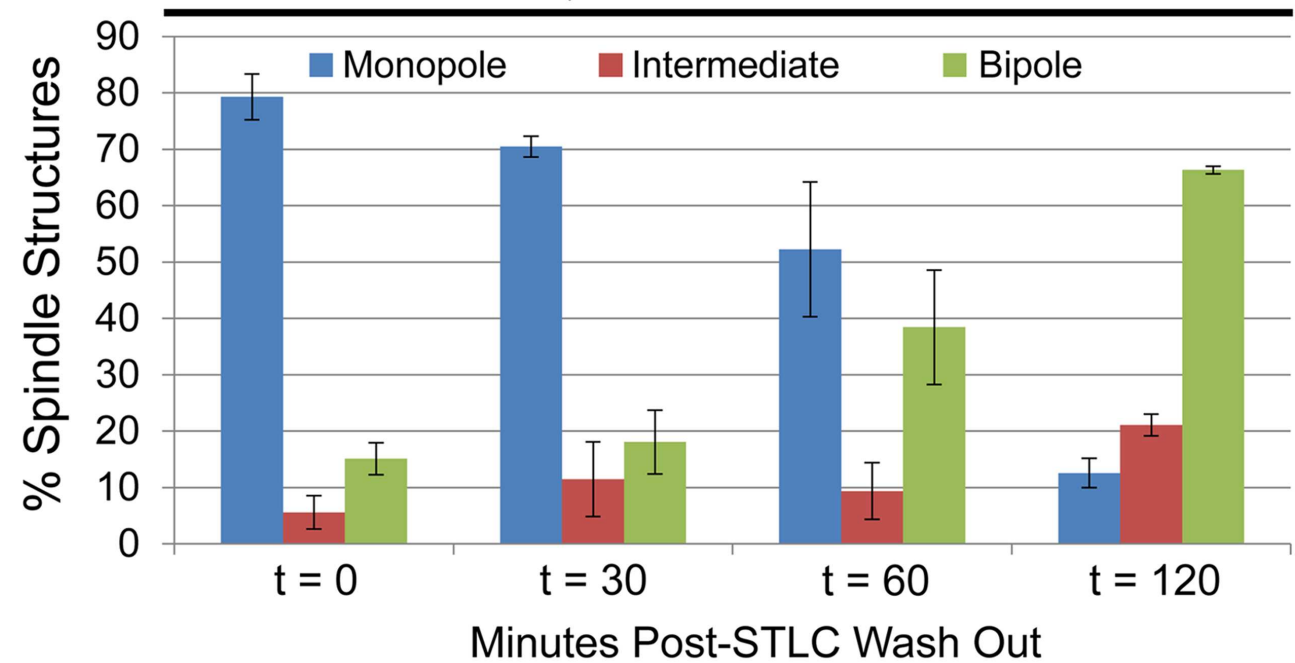

FIGURE 5 | Inhibition of Eg5 with STLC is reversible in RepEg5 cells. (A) Still frames from a time-lapse of a RepEg5 cell before and after the addition of $1 \mu \mathrm{M}$ STLC and following STLC wash out. STLC was added between 0:00 and 2:00 and washed out at 21:44. Addition of STLC causes the RepEg5 bipole to collapse into a monopole within $8 \mathrm{~min}$. The RepEg5 monopole reorganizes into a bipole over $\sim 40$ min following STLC wash out and undergoes a bipolar anaphase $\sim 54$ min after removal of the drug. GFP- $\alpha$-tubulin is shown in green and Eg5-mCherry is shown in red. (B) Quantification of spindle morphologies over time (in minutes) after STLC wash out ( $t=0, n=503$ spindles; $t=30$, $n=305 ; t=60, n=403 ; t=120, n=199)$. The percentage of monopoles decreases and the percentage of bipoles increases over time following removal of STLC. Error bars are SEM. Scale bar is $10 \mu \mathrm{m}$.
Xenopus egg extracts (47). After $\sim 20 \mathrm{~min}$, the STLC-containing media was removed from the imaging chamber and replaced with fresh media lacking drug through three to four rounds of media exchange. In the example shown in Figure 5A, re-association of Eg5-mCherry with the spindle was evident within $20 \mathrm{~min}$ of removing STLC. The monopolar spindle reorganized into a bipolar configuration $50 \mathrm{~min}$ after the wash out and underwent a bipolar anaphase shortly thereafter (Figure 5A; Movie S6 in Supplementary Material).

To confirm the live-cell observations, spindle morphologies in fixed RepEg5 cells were quantified at various time points after wash out (Figure 5B). At $t_{0}$, a vast majority of RepEg5 spindles were monopolar ( $\sim 80 \%)$ and only $13 \%$ of spindles were bipolar. The percentage of cells with bipolar spindles steadily increased over time following removal of STLC until $2 \mathrm{~h}$ after STLC wash out a significant majority (66\%) of mitotic spindles in RepEg5 cells were bipolar (Figure 5B). Together, the fixed and live-cell data confirmed that STLC could in fact be washed out from Drosophila RepEg5 cells and that Eg5-mCherry localized and functioned properly after removal of the inhibitor.

\section{ROBUST ERROR CORRECTION IN RepEg5 CELLS REOUIRES AURORA B KINASE}

By their very nature, monopolar spindles cannot establish proper bioriented kt-MT attachments. Syntelic kt-MT attachments, in which both sister kinetochores attach to the same spindle pole, are prevalent in monopoles (47). Erroneous attachments are not permanent; in fact, cells can correct improper kt-MT attachments, 
in large part, through activity of the centromere-enriched ABK $(11,21,48)$. It was previously observed that the transition from monopole to bipole following Eg5 inhibitor wash out was accompanied by the conversion of syntelic attachments to bioriented attachments in an ABK-dependent manner (21).

In order to validate the utility of RepEg5 cells in assaying error correction, the ability of STLC-treated RepEg5 cells to establish syntelic attachments and subsequently repair them following drug wash out was assessed in control and ABK depleted cells (Figures 6A,C). The outer kinetochore protein Ndc80 and microtubules were stained in RepEg5 cells to evaluate attachment state. Syntelic attachments were characterized by juxtaposed sister kinetochores attached to the same spindle pole while bioriented kinetochores were defined by the clear attachment of sister kinetochores to opposite spindle poles. Similar to other cell types, STLC-treatment in RepEg5 control RNAi cells resulted in elevated levels of syntelic attachments around monopolar spindles ( $\sim 60 \%$ of kt-MT attachments). In control cells, $\sim 90 \%$ of spindles were bipolar and a majority of syntelic attachments were converted to bioriented attachments $(>60 \%)$ $2 \mathrm{~h}$ after washing out STLC. The outcome was dramatically different in ABK depleted cells. First, spindles failed to properly bipolarize $\left(80 \%\right.$ at $t_{0}$ versus $\sim 65 \%$ at $\left.t_{120}\right)$ following STLC wash out in the absence of ABK (Figure 6B). Second, syntelic attachments remained consistently high $(>50 \%)$ for the duration of the STLC wash out experiment in the ABK knockdown cells as only $16 \%$ of kt-MT attachments were bioriented two hours after removal of STLC (Figure 6C). The quantification of attachments includes kinetochores from both bipolar $(\sim 40 \%)$ and monopolar $(\sim 60 \%)$ spindles. We included monopolar spindles in our analyses because, in our hands, ABK depletion from S2 cells leads to a preponderance of monopolar spindles. Thus, ABK activity is required for the conversion of monopolar to bipolar spindles and for efficient error correction after STLC wash out in RepEg5 cells.

\section{DISCUSSION}

In this study, a live-cell error correction assay has been successfully recapitulated in Drosophila S2 cells by functionally replacing Drosophila Klp61F with human Eg5. The error correction assay, which was initially developed in marsupial $\mathrm{PtK}_{2}$ cells (21), requires induction of erroneous attachments and the subsequent resolution of mal-oriented to bioriented kt-MT attachments in cells where kinesin-5 is reversibly inactivated with small molecule inhibitors. The fact that Ptk2 cells have relatively few large chromosomes and that the cells remain flat during mitosis makes them a model cell line for studying spindles, chromosomes, and kt-MT attachments; however, the availability and ease of application of genetic and molecular tools in PtK cells is quite limited. Drosophila S2 cells also have a relatively small number of chromosomes and can be flattened experimentally. Most importantly, there is an incredibly powerful range of experimental tools that can be applied to Drosophila cells including whole-genome RNAi screening. However, the error correction assay could not be applied in Drosophila cells due to the fact that available kinesin-5 inhibitors, including monastrol and STLC, do not inhibit Klp61F activity. This limitation has now been overcome.

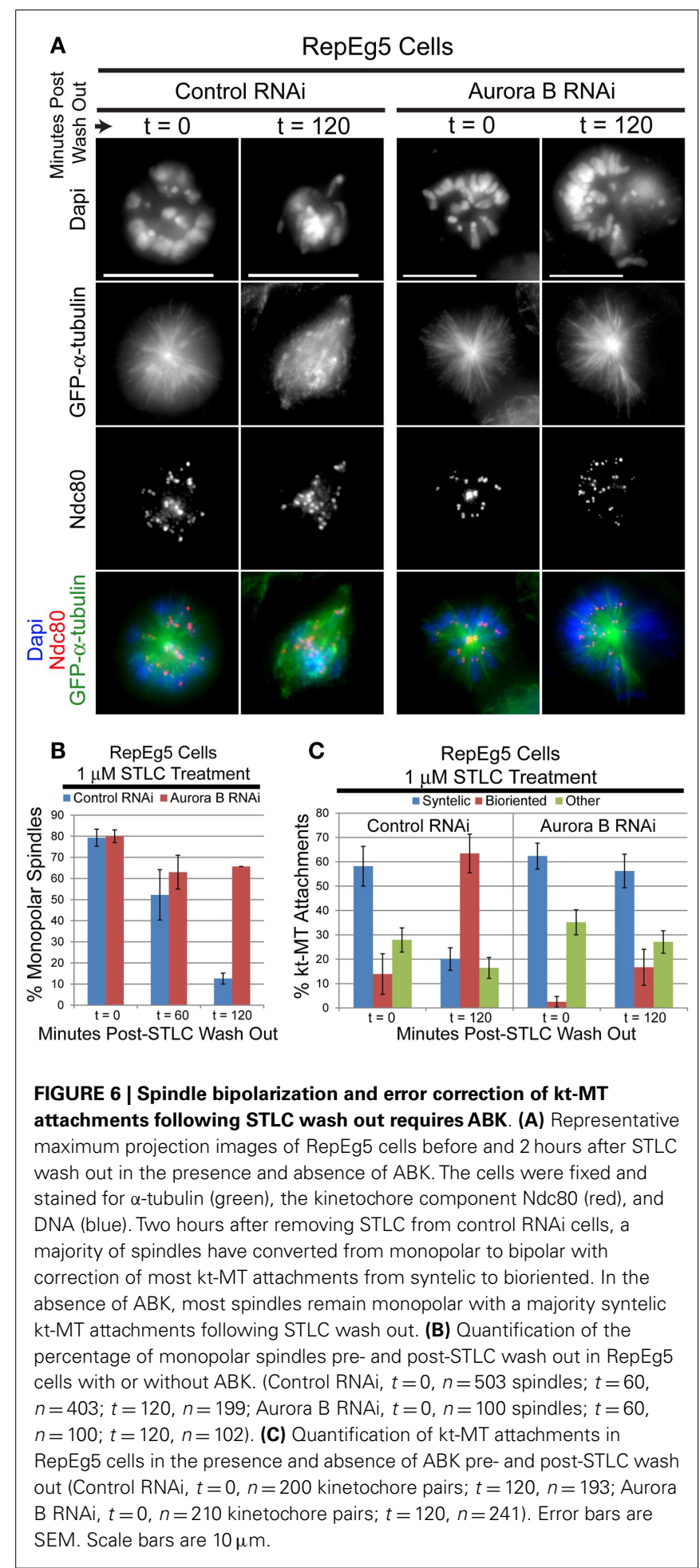

\section{STUDYING THE FUNCTION OF A HUMAN MOTOR IN A FOREIGN CELL TYPE}

At first glance, it may seem non-physiological to examine the function of human Eg5 in an alien cell type. However, the expression of Eg5-mCherry in Drosophila cells has been informative at multiple levels. The fact that human Eg5 rescues Klp61F depletion 
definitively demonstrates that the primary functions of kinesin-5 family members are conserved from fly to man. This is notable because of the fact that many Drosophila genes have rapidly diverged during evolution. In fact, numerous essential cell division regulators, kinetochore components in particular (49), have diverged to such a great extent that Drosophila homologs have been difficult to identify.

The ability of human Eg5 to localize and function normally in fly cells is also revealing about the nature of its regulation. For example, one of the most well-known regulators of Eg5 is the microtubule-associated protein TPX2 $(37,50,51)$. The Drosophila homolog of TPX2 was only recently identified as Ssp1/Mei-38 (now called D-TPX2) (52). Interestingly, D-TPX2 reportedly lacks a highly conserved Eg5-binding domain, which has been shown to be required for proper Eg5 localization and function as well as normal spindle assembly in vertebrate cells $(50,51)$. There are several possible explanations for the discrepancy. First, D-TPX2 may indeed possess a highly diverged Eg5-binding motif that despite lacking any evident sequence similarity to the conserved domain still fulfills the function of interacting with kinesin-5 motors. Second, another Drosophila protein or proteins may fill the role of interacting with and regulating Eg5. Finally, intrinsic characteristics of human Eg5 in combination with organizational properties of the Drosophila spindle may negate the need for TPX2-style regulation of Eg5 in S2 cells.

The findings in RepEg5 cells are also relevant to current spindle assembly models. Balance of force/push-pull models of spindle assembly in vertebrate cells posit that Eg5 is directly antagonized by dynein to establish and maintain spindle bipolarity and spindle length (39-42). However, in Drosophila cells, the force that opposes Klp61F is predominantly generated by the minus-end directed kinesin-14 motor $\operatorname{Ncd}(29,33,34,38,43)$. Interestingly, spindle collapse in RepEg5 cells treated with STLC was largely dependent on Ncd activity. Thus, opposition of human Eg5 can be achieved in a dynein-independent manner. The data suggest that force-balance regulation of human Eg5 depends more on the existence of an opposing force than on the identity of the particular motor that is producing that force.

\section{UPDATING THE FIRST GENERATION DROSOPHILA CELL-BASED ERROR CORRECTION ASSAY TO v2.0}

The work described here has taken an important first step in developing a potentially powerful new cell-based assay for studying error correction during mitosis. However, the first generation RepEg5 cell line has some limitations that could be overcome by creating RepEg5 version 2 (v2.0). First, RepEg5 requires the addition of $\mathrm{CuSO}_{4}$ to the cell media to induce Eg5-mCherry. It would be beneficial to develop cells with constitutive expression of Eg5-mCherry at levels comparable to endogenous Klp61F, which could be achieved by placing expression of Eg5-mCherry under control of the Klp61F promoter. Second, not every cell in the stable population used in this study expressed Eg5 - a property that proved useful in the current study by providing internal controls. However, the combination of a low baseline mitotic index in S2 cells with the fact that $\sim 25-50 \%$ of the cells expressed measurable levels of Eg5-mCherry made quantification time consuming. Thus, it would be useful to generate a clonal population of RepEg5 cells in which every cell expressed identical amounts of Eg5-mCherry at near-endogenous levels. Finally, depletion of Klp61F required a 2-day incubation of RepEg5 cells with exogenous dsRNA with homology to the Klp61F transcript. A stable RepEg5 cell line could be built that incorporates a constitutively expressed short hairpin RNA (shRNA) targeting Klp61F. Thus, RepEg5 v2.0 is envisioned as a clonal population of cells in which Klp61F has truly been replaced with human Eg5 by expressing Eg5-mCherry near-endogenous levels of Klp61F while constitutively blocking production of Klp61F. The RepEg5 v2.0 cell line would be amenable to high-throughput screening assays using STLC and available whole-genome RNAi libraries in combination with algorithms to score spindle morphology in a highly automated manner (24).

Failure to correct erroneous attachments results in CIN and aneuploidy. Aneuploidy in somatic cells has both tumorigenic and tumor protective properties (53) and CIN has been shown to promote tumor adaptation $(54,55)$. Thus, cell-based error correction screens have the potential to identify molecules that could be targeted by drugs in order to modulate CIN.

\section{ACKNOWLEDGMENTS}

We would like to thank Patricia Wadsworth (UMASS Amherst) for generously sharing the anti-Eg5 antibody (Novus Biologicals) and the $H s$ Eg5 DNA construct. We also acknowledge Jonathan Scholey (UC, Davis) for the anti-Klp61F serum. The work was funded in part by Honors Research Grants from the Commonwealth Honors College at UMASS Amherst to Philip T. McGilvray and support from the Charles H. Hood Foundation, Inc., Boston, MA, USA to Thomas J. Maresca and Research Grant No.5-FY13-205 from the March of Dimes Foundation to Thomas J. Maresca.

\section{SUPPLEMENTARY MATERIAL}

The Supplementary Material for this article can be found online at http://www.frontiersin.org/Molecular_and_Cellular_ Oncology/10.3389/fonc.2013.00187/abstract

The Supplementary Material includes six videos and one table.

Movie S1 | Montage of a two-color time-lapse of a Drosophila melanogaster S2 cell expressing GFP- $\alpha$-tubulin (upper left; green in merge) and human Eg5-mCherry (upper right, red in merge) as it progresses through mitosis. Lower left panel is the merged time-lapse. Scale bar is $10 \mu \mathrm{m}$.

Movie S2 | Montage of a two-color time-lapse of a Drosophila melanogaster S2 cell expressing GFP- $\alpha$-tubulin (upper left, green in merge) and human Eg5-mCherry (upper right, red in merge) as it progresses from metaphase through telophase. Lower left panel is the merged time-lapse. Scale bar is $10 \mu \mathrm{m}$.

Movie S3 |Time-lapse of a Drosophila melanogaster S2 cell expressing GFP- $\alpha$-tubulin following addition of $\mathbf{1} \boldsymbol{\mu}$ M STLC. The STLC was added between frames 09:00 and 10:00. Note that the spindle does not collapse into a monopole. Scale bar is $10 \mu \mathrm{m}$.

Movie S4 | Montage of a two-color time-lapse of a Drosophila melanogaster S2 cell expressing GFP- $\alpha$-tubulin (upper left; green in merge) and human Eg5-mCherry (upper right, red in merge) following addition of $1 \mu$ M STLC between frames 0:00 and 02:27. Note that the spindle does not collapse into a monopole despite the fact that spindle-associated levels of Eg5-mCherry drop following addition of the drug. Lower left panel is the merged time-lapse. Scale bar is $10 \mu \mathrm{m}$. 
Movie S5 | Montage of a two-color time-lapse of KIp61F-depleted cells. The RepEg5 S2 cell expressing GFP- $\alpha$-tubulin (upper left; green in merge) and human Eg5-mCherry (upper right, red in merge) is next to a cell that is not expressing Eg5-mCherry. Note that at the beginning of the movie the RepEg5 cell has a bipolar spindle while the nearby cell that is not expressing Eg5 has a monopolar spindle. The bipolar spindle in the RepEg5 cell rapidly collapses into a monopole following the addition of $10 \mu \mathrm{M}$ STLC between frames 00:00 and 02:00 and remains a monopole for the duration of the experiment. Following addition of STLC, spindle-associated levels of Eg5-mCherry drops while cytoplasmic mCherry fluorescence increases. Lower left panel is the merged time-lapse. Scale bar is $10 \mu \mathrm{m}$.

\section{REFERENCES}

1. Rattner JB, Bazett-Jones DP. Kinetochore structure: electron spectroscopic imaging of the kinetochore. J Cell Biol (1989) 108(4):1209-19. doi:10.1083/jcb.108.4.1209

2. Schittenhelm RB, Heeger S, Althoff F, Walter A, Heidmann S, Mechtler K, et al. Spatial organization of a ubiquitous eukaryotic kinetochore protein network in Drosophila chromosomes. Chromosoma (2007) 116(4): 385-402. doi:10.1007/s00412-0070103-y

3. Cheeseman IM, Desai A. Molecular architecture of the kinetochoremicrotubule interface. Nat Rev Mol Cell Biol (2008) 9(1):33-46. doi: $10.1038 / \mathrm{nrm} 2310$

4. Joglekar AP, Bloom K, Salmon ED. In vivo protein architecture of the eukaryotic kinetochore with nanometer scale accuracy. Curr Biol (2009) 19(8):694-9. doi:10.1016/j. cub.2009.02.056

5. Wan X, O'Quinn RP, Pierce HL, Joglekar AP, Gall WE, DeLuca JG, et al. Protein architecture of the human kinetochore microtubule attachment site. Cell (2009) 137(4):672-84. doi: 10.1016/j.cell.2009.03.035

6. Daum JR, Wren JD, Daniel JJ, Sivakumar S, McAvoy JN, Potapova TA, et al. Ska3 is required for spindle checkpoint silencing and the maintenance of chromosome cohesion in mitosis. Curr Biol (2009) 19(17):1467-72. doi:10.1016/j.cub. 2009.07.017

7. Gaitanos TN, Santamaria A, Jeyaprakash AA, Wang B, Conti E, Nigg EA. Stable kinetochoremicrotubule interactions depend on the Ska complex and its new component Ska3/C13Orf3. EMBO J (2009) 28(10):1442-52. doi: 10.1038/emboj.2009.96

8. Raaijmakers JA, Tanenbaum ME, Maia AF, Medema RH. RAMAl is a novel kinetochore protein involved in kinetochoremicrotubule attachment. J Cell Sci (2009) 122(Pt 14):2436-45. doi: $10.1242 /$ jcs. 051912
9. Theis M, Slabicki M, Junqueira M, Paszkowski-Rogacz M, Sontheimer J, Kittler R, et al. Comparative profiling identifies C13orf3 as a component of the Ska complex required for mammalian cell division. $E M B O$ J (2009) 28(10):1453-65. doi:10. 1038/emboj.2009.114

10. Welburn JP, Grishchuk EL Backer CB, Wilson-Kubalek EM, Yates JR3rd, Cheeseman IM. The human kinetochore Skal complex facilitates microtubule depolymerization-coupled motility. Dev Cell (2009) 16(3):374-85. doi: 10.1016/j.devcel.2009.01.011

11. Kitajima TS, Ohsugi M, Ellenberg J. Complete kinetochore tracking reveals error-prone homologous chromosome biorientation in mammalian oocytes. Cell (2011) 146(4):568-81. doi: 10.1016/j.cell.2011.07.031

12. Biggins S, Murray AW. The budding yeast protein kinase Ipll/Aurora allows the absence of tension to activate the spindle checkpoint. Genes Dev (2001) 15(23):3118-29. doi:10. 1101/gad.934801

13. Cheeseman IM, Anderson S, Jwa M, Green EM, Kang Js, Yates JR3rd, et al. Phosphoregulation of kinetochoremicrotubule attachments by the Aurora kinase Ipllp. Cell (2002) 111(2):163-72. doi: 10.1016/S0092-8674(02)00973-X

14. Cheeseman IM, Chappie JS Wilson-Kubalek EM, Desai A. The conserved KMN network constitutes the core microtubulebinding site of the kinetochore. Cell (2006) 127(5):983-97. doi: 10.1016/j.cell.2006.09.039

15. DeLuca JG, Gall WE, Ciferri C, Cimini D, Musacchio A, Salmon ED. Kinetochore microtubule dynamics and attachment stability are regulated by Hecl. Cell (2006) 127(5):969-82. doi: 10.1016/j.cell.2006.09.047

16. Liu D, Vader G, Vromans MJ, Lampson MA, Lens SM. Sensing chromosome bi-orientation by spatial separation of aurora B kinase from kinetochore substrates.

Movie S6 | Montage of a two-color time-lapse of KIp61F-depleted cells. The RepEg5 S2 cell expressing GFP- $\alpha$-tubulin (upper left; green in merge) and human Eg5-mCherry (upper right, red in merge) is next to a cell that is not expressing Eg5-mCherry. Note that at the beginning of the movie the RepEg5 cell has a bipolar spindle while a nearby mitotic cell that is not expressing Eg5 has a monopolar spindle. The bipolar spindle in the RepEg5 cell collapses into a monopole after addition of $1 \mu \mathrm{M}$ STLC between frames 00:00 and 02:00. After washing out the STLC at 21:44 the monopole converts back to a bipole and undergoes anaphase starting at 75:46. Scale bar is $10 \mu \mathrm{m}$.

Table S1 |The primers used in this study. The underlined bases represent the T7 promoter sequence.

Science (2009) 323(5919):1350-3 doi:10.1126/science.1166368

17. Welburn JP, Vleugel M, Liu D, Yates JR3rd, Lampson MA, Fukagawa $\mathrm{T}$, et al. Aurora B phosphorylates spatially distinct targets to differentially regulate the kinetochore-microtubule interface. Mol Cell (2010) 38(3): 383-92. doi:10.1016/j.molcel.2010. 02.034

18. Maresca TJ, Salmon ED. Welcome to a new kind of tension: translating kinetochore mechanics into a waitanaphase signal. J Cell Sci (2010) 123(Pt 6):825-35. doi:10.1242/jcs. 064790

19. Ditchfield C, Johnson VL, Tighe A, Ellston R, Haworth C, Johnson $\mathrm{T}$, et al. Aurora B couples chromosome alignment with anaphase by targeting BubR1, Mad2, and Cenp-E to kinetochores. J Cell Biol (2003) 161(2):267-80. doi:10.1083/ jcb.200208091

20. Hauf S, Cole RW, LaTerra S, Zimmer C, Schnapp G, Walter R, et al. The small molecule Hesperadin reveals a role for Aurora B in correcting kinetochore-microtubule attachment and in maintaining the spindle assembly checkpoint. J Cell Biol (2003) 161(2):281-94. doi: 10.1083/jcb.200208092

21. Lampson MA, Renduchitala $K$ Khodjakov A, Kapoor TM. Correcting improper chromosome-spindle attachments during cell division. Nat Cell Biol (2004) 6(3):232-7. doi: 10.1038/ncb1102

22. Olszak AM, van EssenD, Pereira AJ, Diehl S, Manke T, Maiato $\mathrm{H}$, et al. Heterochromatin boundaries are hotspots for de novo kinetochore formation. Nat Cell Biol (2011) 13(7):799-808. doi:10.1038/ ncb 2272

23. Schneider I. Cell lines derived from late embryonic stages of Drosophila melanogaster. J Embryol Exp Morphol (1972) 27(2):353-65.

24. Goshima G, Wollman R, Goodwin SS, Zhang N, Scholey JM, Vale RD, et al. Genes required for mitotic spindle assembly in Drosophila S2 cells.
Science (2007) 316(5823):417-21. doi:10.1126/science.1141314

25. Perrimon N, Mathey-Prevot B. Applications of highthroughput RNA interference screens to problems in cell and developmental biology. Genetics (2007) 175(1):7-16. doi: 10.1534/genetics.106.069963

26. Maliga Z, Mitchison TJ. Smallmolecule and mutational analysis of allosteric Eg5 inhibition by monastrol. BMC Chem Biol (2006) 6:2. doi:10.1186/1472-6769-6-2

27. Learman SS, Kim CD, Stevens NS, Kim S, Wojcik EJ, Walker RA. NSC 622124 inhibits human Eg5 and other kinesins via interaction with the conserved microtubule-binding site. Biochemistry (2009) 48(8):1754-62. doi: 10.1021/bi801291q

28. Liu L, Parameswaran S, Liu J, Kim S, Wojcik EJ. Loop 5-directed compounds inhibit chimeric kinesin5 motors: implications for conserved allosteric mechanisms. J Biol Chem (2011) 286(8):6201-10. doi: 10.1074/jbc.M110.154989

29. Maresca TJ, Salmon ED. Intrakinetochore stretch is associated with changes in kinetochore phosphorylation and spindle assembly checkpoint activity. J Cell Biol (2009) 184(3):373-81. doi:10.1083/ jcb. 200808130

30. Houliston E, Le GuellecR, Kress M, Philippe M, Le GuellecK. The kinesin-related protein Eg5 associates with both interphase and spindle microtubules during Xenopus early development. Dev Biol (1994) 164(1):147-59. doi:10.1006/ dbio.1994.1187

31. Sawin KE, LeGuellec K, Philippe M, Mitchison TJ. Mitotic spindle organization by a plus-enddirected microtubule motor. Nature (1992) 359(6395):540-3. doi:10. 1038/359540a0

32. Heck MM, Pereira A, Pesavento P, Yannoni Y, Spradling AC, Goldstein LS. The kinesin-like protein KLP61F is essential for mitosis in Drosophila. J Cell Biol (1993) 123(3):665-79. doi:10.1083/jcb.123.3.665 
33. Sharp DJ, Yu KR, Sisson JC, Sullivan W, Scholey JM. Antagonistic microtubule-sliding motors position mitotic centrosomes in Drosophila early embryos. Nat Cell Biol (1999) 1(1):51-4. doi:10.1038/ 9025

34. Goshima G, Vale RD. The roles of microtubule-based motor proteins in mitosis: comprehensive RNAi analysis in the Drosophila S2 cell line. I Cell Biol (2003) 162(6):1003-16. doi: 10.1083/jcb.200303022

35. Mayer TU, Kapoor TM, Haggarty SJ, King RW, Schreiber SL, Mitchison TJ. Small molecule inhibitor of mitotic spindle bipolarity identified in a phenotype-based screen. Science (1999) 286(5441):971-4. doi: 10.1126/science.286.5441.971

36. Skoufias DA, DeBonis S, Saoudi Y, Lebeau L, Crevel I, Cross R, et al. Strityl-L-cysteine is a reversible, tight binding inhibitor of the human kinesin Eg5 that specifically blocks mitotic progression. J Biol Chem (2006) 281(26):17559-69. doi:10. 1074/jbc.M511735200

37. Gable A, Qiu M, Titus J, Balchand S, Ferenz NP, Ma N, et al. Dynamic reorganization of Eg5 in the mammalian spindle throughout mitosis requires dynein and TPX2. Mol Biol Cell (2012) 23(7):1254-66. doi: 10.1091/mbc.E11-09-0820

38. Sharp DJ, Brown HM, Kwon M, Rogers GC, Holland G, Scholey JM. Functional coordination of three mitotic motors in Drosophila embryos. Mol Biol Cell (2000) 11(1): 241-53. doi:10.1091/mbc.11.1.241

39. Gaglio T, Saredi A, Bingham JB, Hasbani MJ, Gill SR, Schroer TA, et al. Opposing motor activities are required for the organization of the mammalian mitotic spindle pole.
J Cell Biol (1996) 135(2):399-414. doi:10.1083/jcb.135.2.399

40. Mitchison TJ, Maddox P, Gaetz J, Groen A, Shirasu M, Desai A, et al. Roles of polymerization dynamics, opposed motors, and a tensile element in governing the length of Xenopus extract meiotic spindles. Mol Biol Cell (2005) 16(6): 3064-76. doi:10.1091/mbc.E05-020174

41. Ferenz NP, Paul R, Fagerstrom C, Mogilner A, Wadsworth P. Dynein antagonizes eg 5 by crosslinking and sliding antiparallel microtubules. Curr Biol (2009) 19(21):1833-8. doi:10.1016/j.cub.2009.09.025

42. Gatlin JC, Matov A, Groen AC, Needleman DJ, Maresca TJ, Danuser $\mathrm{G}$, et al. Spindle fusion requires dynein-mediated sliding of oppositely oriented microtubules. Curr Biol (2009) 19(4):287-96. doi:10. 1016/j.cub.2009.01.055

43. Tao L, Mogilner A, CivelekogluScholey G, Wollman R, Evans J, Stahlberg $\mathrm{H}$, et al. A homotetrameric kinesin-5, KLP61F, bundles microtubules and antagonizes Ncd in motility assays. Curr Biol (2006) 16(23):2293-302. doi: 10.1016/j.cub.2006.09.064

44. Endow SA, Chandra R, Komma DJ, Yamamoto AH, Salmon ED. Mutants of the Drosophila Ncd microtubule motor protein cause centrosomal and spindle pole defects in mitosis. J Cell Sci (1994) 107(Pt 4):859-67.

45. Goshima G, Nedelec F, Vale RD. Mechanisms for focusing mitotic spindle poles by minus enddirected motor proteins. J Cell Biol (2005) 171(2):229-40. doi:10.1083/ jcb.200505107

46. Cameron LA, Yang G, Cimini D, Canman JC, Kisurina-Evgenieva O,
Khodjakov A, et al. Kinesin 5independent poleward flux of kinetochore microtubules in PtK1 cells. J Cell Biol (2006) 173(2):173-9. doi: 10.1083/jcb.200601075

47. Kapoor TM, Mayer TU, Coughlin ML, Mitchison TJ. Probing spindle assembly mechanisms with monastrol, a small molecule inhibitor of the mitotic kinesin, Eg5. J Cell Biol (2000) 150(5):975-88. doi:10.1083/ jcb.150.5.975

48. Cimini D, Wan $\mathrm{X}$, Hirel CB, Salmon ED. Aurora kinase promotes turnover of kinetochore microtubules to reduce chromosome segregation errors. Curr Biol (2006) 16(17):1711-8. doi:10.1016/ j.cub.2006.07.022

49. Przewloka MR, Zhang W, Costa $\mathrm{P}$, Archambault V, D'Avino PP, Lilley KS, et al. Molecular analysis of core kinetochore composition and assembly in Drosophila melanogaster. PLoS ONE (2007) 2(5):e478. doi: 10.1371/journal.pone.0000478

50. Eckerdt F, Eyers PA, Lewellyn AL, Prigent C, Maller JL. Spindle pole regulation by a discrete Eg5interacting domain in TPX2. Curr Biol (2008) 18(7):519-25. doi:10. 1016/j.cub.2008.02.077

51. Ma N, Titus J, Gable A, Ross JL, Wadsworth P. TPX2 regulates the localization and activity of Eg5 in the mammalian mitotic spindle. $J$ Cell Biol (2011) 195(1):87-98. doi: 10.1083/jcb.201106149

52. Goshima G. Identification of a TPX2-like microtubule-associated protein in Drosophila. PLoS ONE (2011) 6(11):e28120. doi:10.1371/ journal.pone.0028120

53. Weaver BA, Silk AD, Montagna C, Verdier-Pinard P, Cleveland DW.
Aneuploidy acts both oncogenically and as a tumor suppressor. Cancer Cell (2007) 11(1):25-36. doi: 10.1016/j.ccr.2006.12.003

54. Bakhoum SF, Genovese G, Compton DA. Deviant kinetochore microtubule dynamics underlie chromosomal instability. Curr Biol (2009) 19(22):1937-42. doi:10.1016/j.cub. 2009.06.039

55. Bakhoum SF, Compton DA. Chromosomal instability and cancer: a complex relationship with therapeutic potential. J Clin Invest (2012) 122(4):1138-43. doi: 10.1172/JCI59954

56. Sharp DJ, Rogers GC, Scholey JM. Microtubule motors in mitosis. Nature (2000) 407(6800):41-7. doi: $10.1038 / 35024000$

Conflict of Interest Statement: The authors declare that the research was conducted in the absence of any commercial or financial relationships that could be construed as a potential conflict of interest.

Received: 29 April 2013; accepted: 04 July 2013; published online: 23 July 2013 Citation: Salemi JD, McGilvray PT and Maresca TJ (2013) Development of a Drosophila cell-based error correction assay. Front. Oncol. 3:187. doi: 10.3389/fonc. 2013.00187

This article was submitted to Frontiers in Molecular and Cellular Oncology, a specialty of Frontiers in Oncology.

Copyright (c) 2013 Salemi, McGilvray and Maresca. This is an open-access article distributed under the terms of the Creative Commons Attribution License, which permits use, distribution and reproduction in other forums, provided the original authors and source are credited and subject to any copyright notice concerning any third-party graphics etc. 